\title{
Surgical management of adenomyoma with hysteroscopy and laparotomy
}

\author{
David Rose $\cdot$ Emmanuel Ofuasia $\cdot$ Uzma Aziz
}

Received: 13 July 2009 /Accepted: 2 September 2009/Published online: 18 September 2009

(C) Springer-Verlag 2009

\begin{abstract}
A nulliparous woman presented with menorrhagia, anemia, and an enlarged uterus. A diagnosis of leiomyoma was made. At surgery, hysteroscopic resection was not possible because of the size of the mass. Laparotomy was performed and the uterus was opened through a "classical incision". Hemostasis was secured with an intrauterine balloon. Histology revealed the mass to be an adenomyoma. Resecting an adenomyoma from the uterus can be complicated. Techniques, such as hysteroscopic examination before laparotomy, using a classical uterine scar, intrauterine balloon, and preoperative gonadotropin-releasing hormone analog may help to minimize the risks.
\end{abstract}

Keywords Menorrhagia $\cdot$ Hysteroscopy $\cdot$ Adenomyoma . Hemostasis

\section{Case}

A 38-years-old nulliparous Afro-Caribbean lady presented to the gynecology clinic with a 5-year history of menorrhagia. At this visit, she complained of recurrence of her heavy bleeding which had become progressively worse. She had tried extensive medical management that failed to control her menorrhagia. In her past gynecological history, she has had a myomectomy to remove multiple fibroids and subsequent hysteroscopic resection of a fibroid polyp. Histolopathology confirmed leiomyoma on both occasions.

D. Rose $(\triangle) \cdot$ E. Ofuasia $\cdot$ U. Aziz

Mayday University Hospital, Women's Health London United

Kingdom,

London, UK

e-mail: docdr69@yahoo.com
At presentation, she was anemic and clinical examination revealed a uterus which was enlarged to 26-weeks gestation. She also complained of persistent discharge, and vaginal examination revealed a fibroid polyp protruding from the cervical os. An ultrasound scan diagnosed a uterine mass consistent with leiomyoma. A magnetic resonance imaging performed showed a fibroid with heterogenous signal and an extensive area of high signal that may represent necrotic or myxoid changes within a fibroid (Fig. 1). Her family was not completed and therefore any intervention would have to preserve her fertility. A two-stage procedure was planned, firstly resection of her submucosal fibroid polyp followed by myomectomy. She was also offered other options including uterine artery embolization but declined.

Her initial surgery involved removal of an $8 \times 9-\mathrm{cm}$ pedunculated fibroid polyp from the endocervix. Histopathological examination confirmed that this was a leiomyoma.

She was pretreated with the gonadotrophin-releasing hormone (GnRH) analog Goserelin, which started 3 months before her planned abdominal surgery. Routine hysteroscopy was performed prior to her planned abdominal myomectomy and a broad base polyp was seen arising from the fundus and posterior uterine wall.

The polyp measured $10 \mathrm{~cm}$ at its distal end and extended to the uterine fundus where it was attached by a broad base. This made hysteroscopic resection impossible. Laparotomy was done via a midline incision. She had multiple adhesions of the anterior abdominal wall to the bowel and the lower part of the uterus. The uterus was globular and soft and enlarged to 28 -weeks gestation. It also contained three subserosal fibroids which were removed. The ovaries and tubes were adherent to the back of the uterus but were freed. The uterus was opened through a midline "classical" incision. Arising from the fundus was a large pedunculated 


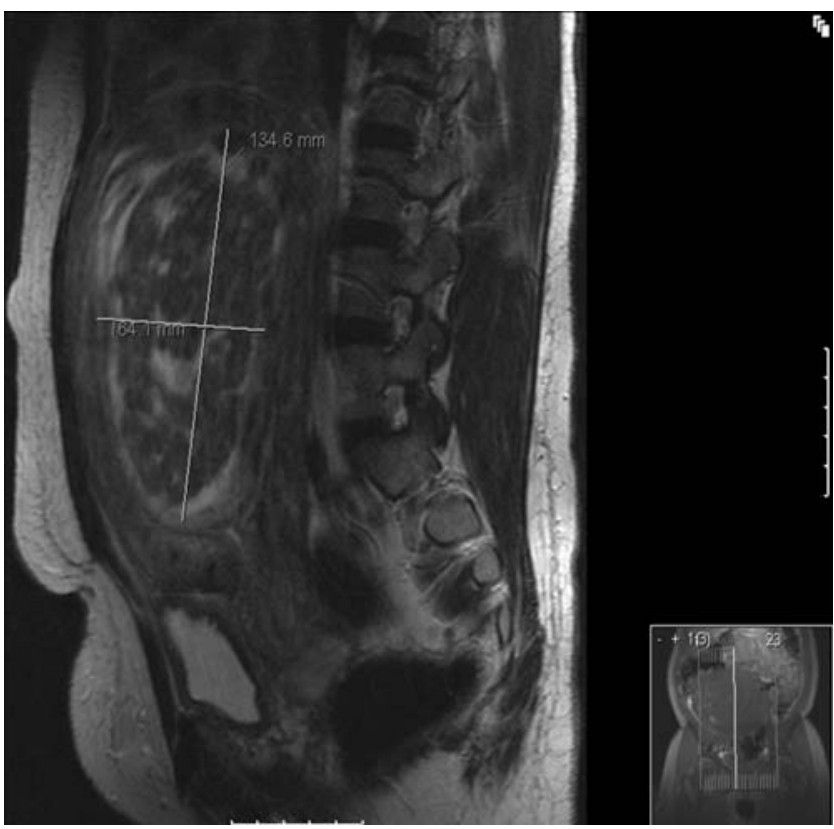

Fig. 1 MRI of uterine tumor

fibroid with a base of $10 \mathrm{~cm}$ and a length of approximately $20 \mathrm{~cm}$ (Fig. 2). The polyp was dissected from its base along a distinct plane. Hemostasis was easily achieved and the endometrial layer apposed. In order to minimize the risk of peritoneal infection, an attempt was made to deliver the specimen vaginally but its broad base restricted its passage through the cervix and thus vaginal delivery failed. The polyp was then delivered intact through the uterine incision. The uterus was then closed in two layers. Postoperative hemostasis was maintained by insertion of a Rusch balloon in uterine cavity before closing the uterus. The balloon was deflated $48 \mathrm{~h}$ later. The estimated intraoperative blood loss was 11 . The patient made a good recovery and was discharged from hospital on the ninth postoperative day. She was advised to delay pregnancy until after her 3-month follow-up appointment. The histology report confirmed that the smaller specimens were indeed fibroids however, the larger polyp was an adenomyoma.

She attended a follow-up appointment and had already achieved two normal periods with no evidence of recurrence of disease. A transvaginal scan was normal. She was discharged with advice to start her family as desired.

\section{Discussion}

Menorrhagia is one of the most common gynecology problems and accounts for $40 \%$ of out-patient consultation [1]. Uterine fibroid remains a common cause of menorrhagia and is the leading indication for surgical intervention. Given the relative frequency with which fibroids are found

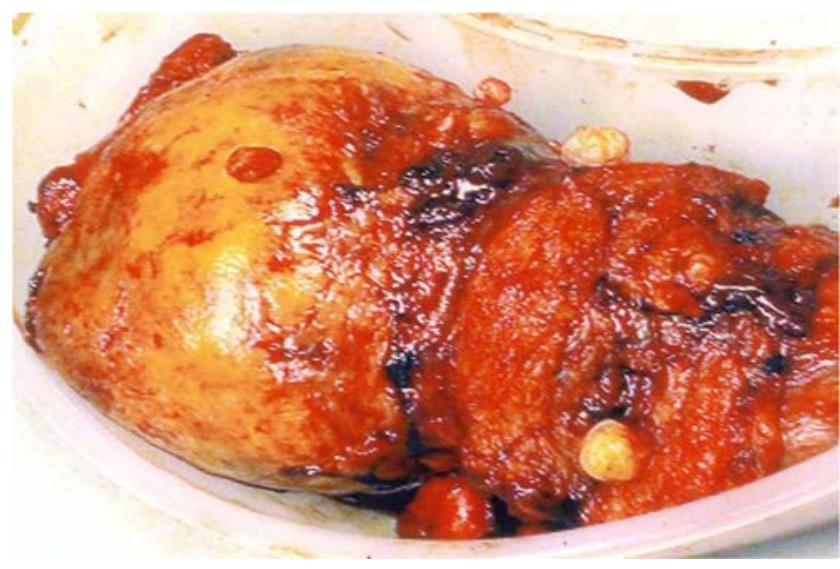

Fig. 2 Excised adenomyoma

in women with menorrhagia and an enlarged uterus, clinically it has to be the diagnosis until proven otherwise, whenever we encounter such women. Our case clearly reiterates the necessity to entertain a differential diagnosis.

Adenomyoma are rare benign tumors of the uterus, occurring predominantly in premenopausal women where preservation of fertility may be an important issue in management. Histologically, they are composed of glands lined by endometrial type epithelium. They are separated by endometrial stroma and leiomyomatous smooth muscle. They may also contained blood-filled cyst, also lined with endometrial epithelium. Microscopic examination helps to differentiate them from other tumors which they may mimic. Leiomyomata have no glandular elements while atypical polypoid adenomyoma display cellular atypia. Adenosarcoma are malignant with mitotic activity in the stroma and smooth muscle. It would appear that malignant transformation is rare with very few cases reported in the literature [2]. This makes conservative management a feasible option.

They are usually confined to the uterus, within the myometrium or a polyp arising from the endometrium. However, there have been reported cases in the broad ligament and ovarian ligament [3]. Comorbidity is common, with fibroids and adenomyosis present in $46.9 \%$ and $30.8 \%$ of cases, respectively [4].

Clinically, patients with adenomyoma present with symptoms synonymous with women who have uterine fibroid, i.e., menorrhagia and enlarged uterus. This makes a diagnosis difficult. Adenomyomas display specific features on imaging. Sonographic characteristics include an isoechoic mass with hemorrhagic foci and poorly defined margins. Doppler studies may show a single vascular pedicle entering the mass [5]. Ultrasound scan has a high negative predictive value $(98.6 \%)$ and can be useful in supporting or refuting the diagnosis [6]. The sensitivity is, however, low when compared to its use in diagnosing fibroids. Magnetic resonance imaging is a useful adjunct with features that aid 
in the diagnosis. Adenomyomata classical appear isointense relative to the myometrium with hyperintense foci on weighted images [7]. However, the signs are subtle and may be missed if the diagnosis is not considered.

Adenomyomata are usually treated by excision or hysterectomy. Hysteroscopic resection of tumors is associated with decrease morbidity and quicker recovery, however, it is a procedure restricted by the size of the mass. Multistage hysteroscopic resection has been used to remove large fibroids but we decided to proceed to laparotomy because of the fact that our patient had already had several surgical excisions and the rapid regrowth of her tumor. The fact that the mass was an adenomyoma may well explain the recurrence of such a large mass over such a short period, which is uncharacteristic of fibroids.

Hemostasis was a concern, and steps were taken to minimize blood loss. Firstly, she was pretreated with $\mathrm{GnRH}$ analog. This has been shown to decrease blood loss in surgical management of fibroids. There is little record of its use in adenomyoma but the effect should be similar since analogs cause myometrial contraction and shrinkage of estrogen-dependent tumors. Surgery combined with postoperative analog treatment appear to be effective in reducing relapse of symptoms and may be appropriate in women willing to postpone their efforts to achieve conception [8]. The use of a hemostatic balloon is common in obstetric hemorrhage. There is also an increasing use of balloons (and Foley's catheter) in hysteroscopic surgery as well as cervical and scar pregnancies to control bleeding [9, 10]. Because these devices provide tamponade within the cavity, they can provide effective hemostasis in cases where tumors or polyps are removed from the endometrial cavity.

Fertility is a major issue in this group. There is some evidence to suggest that the presence of an adenomyoma impacts on fertility [11], although data from large studies is not available. In a study specifically addressing fertility after conservative surgery, $72 \%$ of women conceived following adenomyomectomy, although only $50 \%$ of these progressed to term [12].

In any extensive uterine surgery, the risks in consequent pregnancies must be considered. Mode of delivery was discussed extensively with our patient. Given the extent of uterine surgery, early elective cesarean section has been recommended and we suggest abdominal delivery even if preterm as long as viability has been achieved. This is consistent with current recommendations following similar surgery for other benign uterine tumors.
Follow-up is important in this group. There was no clear indication for a repeat hysteroscopy. This is because the tumor had been easily separated from the uterus and a transvaginal scan was normal. Furthermore, functional integrity had been demonstrated. However, in patients whose surgery significantly disrupted the endometrium, integrity can be checked with out-patient hysteroscopy or sonographic techniques.

Conflict of interest There is no actual or potential conflict of interest in relation to this article.

\section{References}

1. Royal College of Obstetricians and Gynecologists (2004) RCOG national evidence-based clinical guidelines on management of menorrhagia in secondary care. RCOG, London

2. Bockage T, Lee KR, Belinson JL (1992) Uterine mullerian adenosarcoma following adenomyoma in a woman on tamoxifen therapy. Gynecol Oncol 44(1):104-109

3. Choudrie L, Mahajan NN, Solomon MV, Thomas A, Kale AJ, Mahogan K (2007) Ovarian ligament adenomyona: a case report. Acta Chir Belg 107(1):84-85

4. Tahlan A, Nanda A, Mohan H (2006) Uterine Adenomyoma: a clinicopathologic review of 26 cases and a review of the literature. Int J Gynecol Pathol 25(4):361-365

5. Lee EJ, Han JH, Rvu HS (2004) Polypoid adenomyomas: sonohysterographic and colour Doppler findings with histopathologic correlation. J Ultrasound Med 23(11):1421-1429

6. Fedele L, Bianchi J, Dorta M, Zanotti F, Brioschi D, Carinelli S (1992) Transvaginal ultrasonography in differential diagnosis of adenomyoma versus leiomyoma. Am J Obstet Gynecol 167 (3):603-606

7. Kitajima K, Imanaka K, Kuwata Y, Hashimoto K, Sugimura K (2007) MRI of typical polypoid adenomyoma of the uterus in 8 patients: correlation with pathologic findings. J Comput Assist Tomogr 31(3):463-468

8. Wang PH, Liu WM, Fuh JL, Cheng MH, Chao HT (2008) Comparison of surgery alone and combined surgical-medical treatment in the management of symptomatic uterine adenomyoma. Fertil Steril. 92(3):876-885

9. Molinaro T, Barnhart K (2007) Ectopic pregnancies in unusual locations. Seminars in Reproductive Medicine 25(2):123-130

10. Pasini A, Belloni C (2001) Intraoperative complications of 697 consecutive operative hysteroscopies. Minerva Ginecol 53 (1): $13-20$

11. Wang JH, He XH, Wu RJ, Xu XR (2007) Clinicopathologic characteristics of uterine adenomyoma in pregnant women. Fertil Steril 88(1):172-175

12. Fedele L, Bianchi S, Zanotti F, Marchini M, Candiani GB (1993) Fertility after conservative surgery for adenomyomas. Hum Reprod 8(10):1708-1710 